\title{
Biotecnologia de embriões: III - Otimização do momento da inseminação artificial em fêmeas bovinas superovuladas, para programas de transferência de embriões.
}

\author{
Embryos' Biotecnology: III - Optimizing of the Moment of Artificial \\ Insemination in Superovulated Cows in Embryos Transfer Programs.
}

\author{
Marcio Ricardo Costa dos Santos'; Rita Leal Paixão²; \\ Carlos Fernando Marins Rodrigues ${ }^{3}$
}

\section{Resumo}

O objetivo do presente trabalho foi comparar diversos esquemas de inseminação artificial (IA) utilizados em fêmeas bovinas de diferentes raças, doadoras de embriões, de forma a identificar o momento correto da inseminação em programas de transferência de embriões. Computou-se um total de 101 tratamento de indução à superovulação, com FSH-P e sincronização do estro pela aplicação da $\mathrm{PGF}_{2_{\alpha}}$. Os animais foram divididos em quatro grupos para estabelecer-se os esquemas da IA. A partir da detecção do estro, dois grupos de fêmeas sofreram três inseminações com $12 \mathrm{~h}$ de intervalo (grupo 1) ou duas inseminações com $12 \mathrm{~h}$ de intervalo (grupo 2), respectivamente esquema 1 e 2. Nos outros dois grupos, onde não se considerou a detecção do estro, as fêmeas sofreram três inseminações, 48, 60 e $72 \mathrm{~h}$ após a aplicação do agente luteolítico (grupo 3), ou duas inseminações, 48 e $60 \mathrm{~h}$ após a aplicação do mesmo agente (grupo 4), o que caracterizou os esquemas 3 e 4 , respectivamente.

A análise das estruturas embrionárias coletadas no $7^{\circ}$ dia após a IA, demonstrou não haver diferença significativa $(P>0.05)$ entre os quatro esquemas de inseminação utilizados, em relação ao número total de embriões recuperados, ao número de embriões transferíveis, ao percentual de embriões degenerados, de não fecundados e de transferíveis. Esses achados indicam que, não foram necessárias mais do que duas inseminações nas fêmeas doadoras superovuladas, para obter-se até $54,98 \pm 35,26 \%$ de embriões transferíveis, e que o momento da aplicação da PGF ${ }_{2 \alpha}$ pode ser utilizado como um parâmetro confiável, para determinar-se o momento correto da Inseminação Artificial.

Palavras-chave: transferência de embriões, inseminação artificial, bovinos, superovulação.

\section{Introdução}

Atualmente, a técnica de transferência de embriões, combinada à superovulação da doadora, objetiva alcançar número cada vez maiores de embriões transferíveis. Um número maior de produtos pode ser alcançado através do aumento de ovulações, sem diminuir o percentual de fecundação e o desenvolvimento embrionário, ou através do aumento de embriões viáveis com a mesma taxa de ovulação (Foote \& Ellington, 1988). O presente trabalho visa destacar os fatores que influenciam na taxa de fecundação e subseqüente produção de embriões viáveis, com enfoque na importância de otimização do momento da IA. Em fêmeas superovuladas, o momento preciso da $I A$, e o número de espermatozóides morfologicamente normais no local de fecundação são fatores fundamentais para a otimização das taxas de fecundação (Schiewe et al., 1987). Para Hawk et al. (1988), a ausência de espermatozóides acessórios em vários oócitos, fecundados ou não, em fêmeas superovuladas, sugere que o número de espermatozóides no local de fecundação é usualmente baixo.

Schiewe et al. (1987) avaliaram a taxa de fecundação e de recuperação embrionária após a utilização de cinco esquemas diferentes de IA. As IA foram realizadas 12, 24, 36 e 48h após o início do estro, nos respectivos grupos 1, 2, 3 e 4, e ainda em todos esses momentos no grupo 5 (controle) foram utilizadas duas doses de sêmen. Seus resultados indicaram maiores percentuais de fecundação e de embriões transferíveis com a utilização de duas doses de sêmen $24 \mathrm{~h}$ após o início do estro, embora, o mesmo autor em outro trabalho (Schiewe et al., 1985), com apenas uma dose de sêmen $24 \mathrm{~h}$ após o início do estro, já tivesse obtido resultados similares. Apenas uma IA, $12 \mathrm{~h}$ após a detecção do estro, é indicada também por Critser et al. (1980) e Donaldson (1985). Outros autores indicam

\footnotetext{
- Projeto com financiamento do CNPq/FINEP.

1 - Med. Vet. Prof. Dr. Depto. Patologia Clínica - VET. - UFF. Pesq. do CNPq. Cx. Postal 100086 - CEP 24230-340 - Niterói - R.J.

2 - Med. Vet. Prof. MS. Dpto. Fisiologia - CMB - UFF. Cx. Postal 100086 - CEP 24230-340 - Niterói - R.J.

3 -. Med. Vet. Gertec Embriões. Cx. Postal 548 - CEP 12940-000 - Atibaia - S.P.
} 
um esquema de duas inseminações, sendo a primeira $12 \mathrm{~h}$ e a segunda $24 \mathrm{~h}$ após a detecção do estro (Hasler et al., 1983; West et al., 1984). Para Szechy (1992), que comparou os seguintes esquemas (1: duas I.A. 12 e 24h após o início do cio; 2 : duas IA com 10 a $12 \mathrm{~h}$ de intervalo entre si. sendo a primeira durante o cio; 3 : três IA, sendo a primeira no cio e as demais 10 e $20 \mathrm{~h}$ depois; e 4: monta natural) a melhor média de fecundação ocorreu quando a primeira inseminação foi realizada durante o cio e uma segunda 10 a $12 \mathrm{~h}$ depois (tipo 2). Já autores como Guibault et al. (1988) e Shallenberger et al. (1988) utilizaram outro esquema de IA em fêmeas superovuladas, baseados no momento da aplicação do agente luteolítico, sendo a primeira $12 \mathrm{~h}$, a segunda $48 \mathrm{~h}$ e terceira $60 \mathrm{~h}$, após a aplicação dâ PGF ${ }_{2 \alpha}$. Ainda tendo como parâmetro a aplicação do agente luteolítico, observa-se que outros autores (Bak et al., 1989; Hackett et al., 1993; Adams et al., 1994) utilizaram, em experimentos diversos, esquema de duas inseminações artificiais, 60 e 72h após a aplicação da $\mathrm{PGF}_{2{ }_{2}}$. independente da observação do estro. Donaldson (1985) não encontrou diferenças com a utilização de uma a quatro doses de sêmen, e concluiu que apenas uma dose era satisfatória, o que é concordante com os achados de West et al. (1984) e Schiewe et al. (1987).

A qualidade do sêmen utilizado influenciou diretamente no percentual de fecundação, reduzindo-o quando foi utilizado sêmen de média ou baixa qualidade para o procedimento da IA em fêmeas superovuladas, segundo vários autores (Callanghan \& King, 1980; Miller et al., 1981). Os resultados de Schiewe et al. (1987) também indicaram a importância da qualidade de sêmen em relação ao percentual de embriões degenerados: "Quando consideramos a importância econômica do número de embriões transferíveis por doadora, em sêmen congelado de alta qualidade deve ser sempre utilizado preferencialmente ao sêmen de qualidade inferior."

\section{Material e Métodos}

Utilizou-se 60 fêmeas bovinas das raças Simental (52), Limousin (3), Parda Suiça (2) e Nelore (3) selecionadas para serem doadoras em programas de trañsferência de embriões, na Gertec Embriões Atibaia, Estado de São Paulo. Algumas fêmeas foram submetidas, mais de uma vez, ao tratamento superovulatório, perfazendo um total de 101 tratamento. No início do experimento, as fêmeas estavam em excelente condição física, não eram lactantes, já tinham apresentado pelo menos dois ciclos estrais regulares após o parto, ou após o último tratamento de superovulação, e apresentavam faixas etárias variadas, entre 3 e 12 anos de idade.
O início do tratamento de superovulação das fêmeas (doadoras), variou entre o $10^{\circ}$ e $12^{\circ}$ dia do ciclo estral, considerando o estro como o dia 0 . $O$ tratamento de estimulação ovárica consistiu na administração de $32 \mathrm{mg}$ de FSH-P (Lab. Schering, U.S.A.) em duas aplicações diárias, por via intramuscular, durante quatro dias consecutivos, em doses decrescentes do 1 \% para $04^{\circ}$ dia de tratamento. Para a sincronização, $48 \mathrm{~h}$ após o início do tratamento superovulatório, as fêmeas receberam a primeira aplicação do agente luteolítico, cloprostenol (Lutalyse - Upjohn Ltd.) $25 \mathrm{mg}$ ou dinoprost (Ciosin - Lab. Coopers) $500_{\mu} \mathrm{g}$, e a segunda aplicação ocorreu $12 \mathrm{~h}$ após a primeira. Para a realização da IA, os animais foram agrupados em quatro esquemas diferentes, de acordo com a observação ou não da manifestação do estro.

Nos animais, em que a observação da manifestação do estro foi utilizada como parâmetro, utilizou-se os dois esquemas a seguir:

- Esquema 1: três inseminações com 12h de intervalo entre as mesmas, após a detecção do estro. Foram analisados 26 tratamentos nesse esquema.

- Esquema 2: duas inseminações com $12 \mathrm{~h}$ de intervalo entre as mesmas, após a detecção do estro. Foram analisados 20 tratamentos nesse esquema.

Nos animais, em que se efetuou a inseminação, independente da observação do estro, utilizaram-se os dois esquemas a seguir:

- Esquema 3: três inseminações, 48, 60 e 72h após a primeira aplicação da $\mathrm{PGF}_{2_{\alpha}}$. Foram analisados 30 tratamentos nesse esquema.

- Esquema 4: duas inseminações, 48 e 60h após a primeira aplicação da $\mathrm{PGG}_{2_{\alpha}}$. Foram analisados 25 tratamentos nesse esquema.

Para detectar-se o estro nos grupos 1 e 2 estabeleceu-se uma rotina de observação visual diária, pela manhã e a tarde, com duração mínima de 30 minutos em cada período, para avaliar as fêmeas que apresentavam manifestações psíquicas de cio. O sêmen utilizado foi adquirido de central de IA. Previamente à inseminação, foram avaliadas a motilidade e o percentual de espermatozóides vivos, utilizando-se uma única dose de $0.5 \mathrm{ml}$ (mín. $20 \times 10^{6} \mathrm{sptz}$ ) em cada inseminação. No $7^{\circ}$ dia após a inseminação, foi realizada a coleta não cirúrgica para a avaliação das estruturas. Antes da lavagem uterina, realizou-se a avaliação dos ovários, através de palpação retal e exame de ultrassonografia, para constatação dos corpos lúteos. Para a lavagem uterina utilizou-se o meio PBSDulbecco's modificado, a $37^{\circ} \mathrm{C}$, e catéter de Foley introduzido no útero. Os lavados foram recolhidos no Encon Filter (Encon, U.S.A.) e distribuídos em cápsulas de evaporação, para a localização dos embriões com um estereomicroscópio. Cada estrutura identificada foi aspirada por uma pipeta Pasteur e transferida para o PBS, contendo $10 \%$ de soro fetal 
bovino, em mini placas de petri descartáveis, a fim de serem submetidos à avaliação com o auxílio de um estereomicroscópico de ótica simples (100 e 400x).

As estruturas coletadas foram classificadas em embriões transferíveis ou degenerados e não fecundados. Desta forma utilizaram-se os seguintes dados para a análise: número total de embriões recuperados, número de embriões transferíveis, percentual de embriões degenerados, percentual de não fecundados e percentual de embriões transferíveis, levando-se em conta os critérios de Kusan (1986) para a determinação dos transferíveis e não transferíveis.

A partir dos dados obtidos, utilizou-se o statiscal analysis system (SAS) para a análise de variância entre os tratamento de IA (esquemas 1, 2, 3 e 4), e a para a comparação de significância entre as médias (Teste de Duncan), tendo sido adotado o método não protegido. O nível de significância estabelecido no presente estudo foi de $5 \%$.

\section{Resultados}

Os 101 tratamentos do programa de transferência de embriões resultaram em diferentes graus de resposta ovariana, do número e da qualidade dos embriões, com o mínimo de uma, e o máximo de 48 estruturas obtidas por coleta. Os resultados da análise das estruturas recuperadas durante o experimento, segundo a qualidade e a quantidade dos embriões (número total de embriões, número de embriões transferíveis, percentual de embriões degenerados, percentual de não fecundados e percentual de transferíveis), e os esquemas de IA utilizados, podem ser observados na Tabela e na Figura. A análise de variância dos parâmetros utilizados não mostrou diferença $(p>0.05)$ para os esquemas de IA. Os procedimentos de comparação das médias através do teste de Duncan, também demonstrou não existir diferença entre os esquemas utilizados de IA.

Tabela: Número e percentual médio de embriões recuperados de vacas superovuladas, segundo a classificação e o esquema de Inseminação Artificial (I.A.).

\section{Discussão}

Os percentuais médios de embriões transferíveis obtidos neste experimento, apresentaram valores abaixo de $60 \%$, de acordo com as observações de Hahn (1983) e de Benevides Filho (1989). Este fato pode em parte ser explicado pela utilização de FSH-P não purificado, já que o conteúdo de LH acarreta um efeito prejudicial ao sincronismo dos gametas, refletindo-se na fecundação e na viabilidade dos embriões (Donaldson et al., 1986; Callensen et al., 1987; Foote \& Ellington, 1988; Herrier et al., 1988; Yamamoto et al., 1993).

Os resultados encontrados em relação aos embriões degenerados e estruturas não fecundadas são explicados pelo fato de que o tratamento superovulatório causa consideráveis modificações no perfil endocrino das doadoras (Greve et al., 1983; Greve et al. 1984; Callensen et al., 1986; Hyttel et al., 1986; Britt \& Holt, 1988; Moraes et al., 1992), com conseqüente influência na qualidade dos gametas, no fenômenos da fecundação e nos embriões (Elsden et al., 1976; Crisman et al., 1980; Greve et al., 1983; Donaldson et al., 1986; Britt et al., 1988; Hawk, 1988).

A análise estatística dos resultados obtidos demonstrou que não houve diferenças significativas $(P>0.05)$ no percentual médio de embriões transferíveis, de embriões degenerados e não fecundados nos quatro esquemas de inseminação artificial utilizados. Estes resultados concordam com Schiewe et al. (1987), que também não obtiveram melhores resultados com três inseminação ao invés de duas. Para o procedimento de cada inseminação, utilizou-se uma única dose de sêmen $\left(20 \times 10^{6} \mathrm{sptz} /\right.$ dose $)$, de acordo com os trabaIhos de Critser et al. (1980), West et al (1984), Donaldson (1985) e Schiewe et al. (1987). O sêmen utilizado era de boa qualidade com fertilidade já evidenciada, dada a importância de tal fator (Ayalon, 1978; Callanghan \& King, 1980; Miller et al., 1981; Schiewe et al., 1987), embora não fosse de apenas um único touro, o que poderia acarretar influência do fator individualidade, segundo Shi et al. (1981), mas o sêmen dos mesmos touros foram utilizados nos quatro esque-

\begin{tabular}{llllll}
\hline $\begin{array}{l}\text { Esquema } \\
\text { de IA }\end{array}$ & $\begin{array}{l}\text { Total de } \\
\text { embriões }(x \pm s)\end{array}$ & $\begin{array}{l}\text { Total de TR } \\
(x \pm s)\end{array}$ & $\begin{array}{l}\% \text { TR } \\
(x \pm s)\end{array}$ & $\begin{array}{l}\% \text { DG } \\
(x \pm s)\end{array}$ & $\begin{array}{l}\% \text { NF } \\
(x \pm s)\end{array}$ \\
\hline 1 & $13,42 \pm 9,21$ & $6,61 \pm 5,77$ & $49,57 \pm 30,17$ & $24,69 \pm 28,40$ & $24,96 \pm 30,94$ \\
\cline { 2 - 6 } 2 & $13,15 \pm 10,24$ & $6,15 \pm 6,32$ & $44,19 \pm 33,46$ & $20,65 \pm 16,99$ & $35,15 \pm 38,47$ \\
\cline { 2 - 6 } 4 & $13,93 \pm 7,89$ & $7,63 \pm 6,26$ & $51,40 \pm 35,84$ & $24,08 \pm 26,42$ & $21,17 \pm 30,65$ \\
\hline
\end{tabular}

$(x \pm s)$ : média e desvio padrão; TR: total de embriões transferíveis; DG: embriões degenerados; NF: percentual de oocitos não fecundados. 


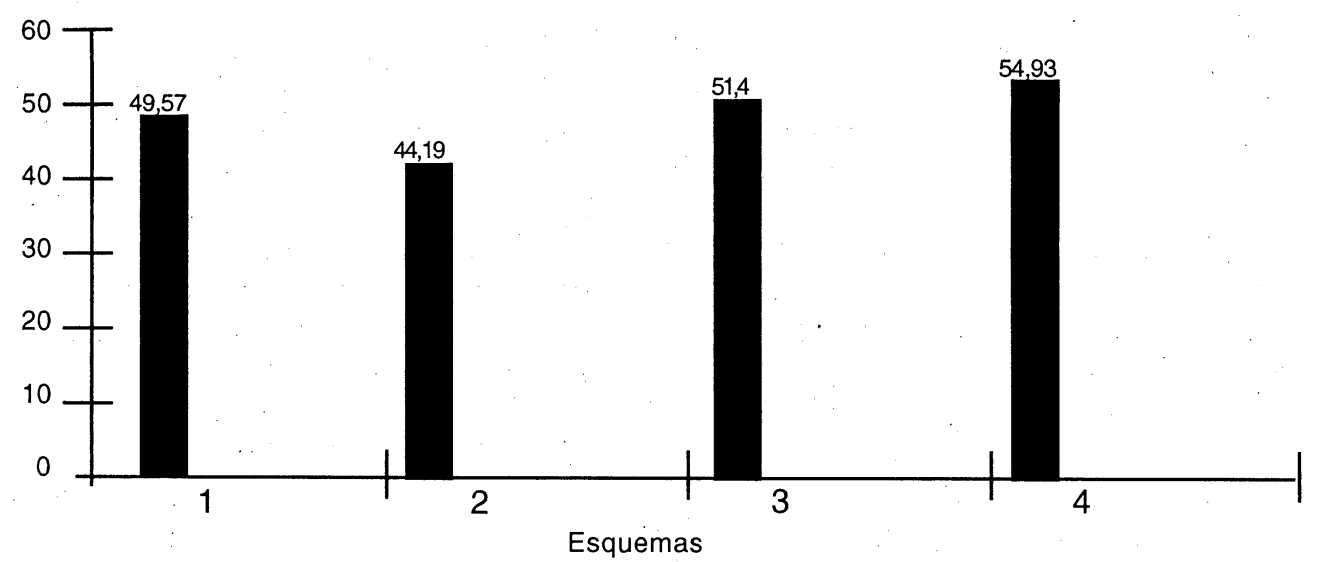

TR: embriões transferiveis

Figura - Percentual médio do número de embriões transferíveis nos esquemas testados.

mas, visando minimizar tais diferenças. Em relação ao parâmetro utilizado, para se determinar o momento da IA, através da detecção do estro ou da aplicação da $\mathrm{PGF}_{2_{\alpha}}$, também não foram observadas diferenças significativas, o que nos permite concordar com a indicação de Guibault et al. (1988) e Schallenberger et al. (1988). Embora as características de estro não se alterem abrigatoriamente com 0 tratamento superovulatório (Coe \& Allrich, 1989), a utilização do momento de aplicação da $\mathrm{PGF}_{2 \alpha}$. diminuiu os transtornos da observação do estro, uma vez que a detecção de seu momento inicial geralmente não ocorre (Hunter, 1986), e quanto mais tardia a inseminação em relação ao início do estro, maior o percentual de embriões degenerados conforme citações de Schiewe et al. (1987), possivelmente devido ao envelhecimento gamético associado a distúrbios como a poliespermia ou alterações cromossômicas (Dalla Porta \& De Bem, 1982; Hunter, 1985; Xu \& Greve, 1988; Benevides Filho \& Pinheiro, 1988). Para Bernard et al. (1984) o próprio início do estro não demonstrou ser um ponto de referência confiável para se avaliar o momento da ovulação, e os estudos de vários autores sobre o momento da ovulação (Christenson et al., 1975; Schams et al., 1977; Maxwell et al., 1978; Shea, 1978; Callensen et al., 1986; Hunter, 1986; Looney., 1986; Yadav et al., 1986; Hyttel et al., 1989) demonstraram também variação do momento da ovulação em relação ao pico de LH e ao momento da aplicação da $\mathrm{PGF}_{2 \alpha}$. além de se considerar o tempo de duração da ovulação em casos de fêmeas superovuladas, e ainda a influência do ritmo circadiano, segundo Schams et al. (1977).

\section{Conclusões:}

Em face dos resultados obtidos no desenvolvimento deste trabalho, em fêmea bovinas superovuladas, pode-se concluir que:

- o momento da aplicação da $\mathrm{PGF}_{2_{\alpha}}$. pode ser considerado como um parâmetro confiável e simplificado para se estabelecer o momento da IA.

- a utilização do momento da aplicação da $\mathrm{PGF}_{2 \alpha}$. como parâmetro minimizar os transtornos de observação do estro.

- os períodos de 48 e 60 h após a aplicação da PGF ${ }_{2 \alpha}$. correspondem aos momentos propícios para a realização da IA.

- a utilização de mais do que duas IA em fêmeas superovuladas é desnecessária em programas de transferência de embriões.

\section{Abstract \\ Embryos' Biotecnology: III - Optimizing of the Moment of Artificial Insemination in Superovulated Cows in Embryos Transfer Programs}

The aim of this study was to compare differents artificial insemination (AI) scheduoles used in donors cows, in order to get the optimun moment of insemination in embryo transfer programs. It was done 101 treatments of induction to superovulation with $\mathrm{FSH}$ $P$ and estrus sincronization with $P G F_{2_{\alpha}}$. The females were divided in four groups in order to establish artificial insemination schedules. From estrus detection, two groups of donor females were inseminated three times (group 1) or twice (group 2) at 12th intervals, respectively schedule 1 and 2 . In the others two groups wich the estrus detection was not considered, the donors were inseminated at 48,60 and $72 \mathrm{~h}$ (group 3 ) or twice, at 48 and 60h (group 4), after luteolitic agent administration, respectively schedules 3 and 4 .

The statistical analysis of recovered strutuctures on day 7 after Al showed no significant differences $(P>0.05)$ in the four schedules in relation to total number of recovered embryos, transferable embryo number, degenerated embryos, no fertilized and transferable embryo rate. These results indicated that only two A.I. were enough to obtain $54.98 \pm 35.26 \%$ of transferable embryos and the moment of $\mathrm{PGF}_{2_{\alpha}}$. administration may 
be used as a trustworthy parameter in order to establish the good time for artificial insemination on cattle.

Key words: embryo transfer, artificial insemination, cattle, superovulation.

\section{Referências Bibliográficas}

ADAMS, G. D.; BO, G. A.; MARTINEZ, M.; CACCIA, M.; TRIBULO, H.; PIERSON, R.A.; MAPLETOFT, R.J. The effect of estradiol-17b and progestogen treatment on superovulatory reponse in beef cows. Theriogenology, v.41, n.1, p.153, 1994.

AYALON, N. A review of embryonic mortality in cattle. J. Reprod. Fert., v.54, p.483-493, 1978. BAK, A.; GREVE, T.; SCHMIDT. Effect of superovulation on reproduction. Theriogenology, 'v. 31, n.1, p.169, 1989.

BENEVIDES FILHO, J. M. Mortalidade embrionária em programas de transferência de embriões. In: SEMANA DE ZOOTECNICA DA FACULDADE DE MEDICINA VETERINÁRIA E ZOOTECNIA DA USP, 134., 1989, Pirassununga. Anais., 1989, p.9-25.

BENEVIDES FILHO, J. M. \& PINHEIRO, L.E.L. Citogenetic analysis of 39 bovine embryos obtained from superovulated females. Rev. Brasil. Genet., v.11, n.3, p.661-670, 1988.

BERNARD, C.; VALET, J.P.; BÉLAND, R.; LAMBERT, R. D. Prédction de l'ovulation par laparoscopie. Can. J. Comp. Med., v.48, p,97-101, 1984.

BRITT, J. H. \& HOLT, L. C. Endocrinological screening of embryo donors and embryo transfer recipients: a review of research with cattle. Theriogenology, v.29, n.1, p.189-202, 1988.

CALLANGHAN, B. D. \& KING, G. J. Determination of the fertilization rate of A.I. sires. Theriogenology, v.14, n.6, p.403-410, 1980.

CALLENSEN, H.; GREVE, T.; HYTTEL, P. Preovulatory endocrinology and oocyte maturation in superovulated cattle. Theriogenology, v.25, n.1, p.71-86, 1986.

CALLENSEN, H.; GREVE, T.; HYTTEL, P. Premature ovulations in superovulated cattle. Theriogenology, v.28, n.2, p.155-166, 1987.

COE, B. L. \& ALLRICH R. D. Relationship between endogenous estradiol - 17 beta and estrous behavior in heifers. J. Anim. Sci., v.67, n.6, p.1546-1551, 1989.

CHRISTENSON, R. K.; ECHTERNKAMP, S. E.; LASTER, D. B. Oestrus, LH, ovulation and fertility in beef heifers. J. Reprod. Fertil., v.43, p.543-546, 1975.
CRISMAM, R. O.; MCDONALD, L. E.; THOMPSON, F.N. Effects of progesterone or estradiol on uterine tubal transport of ova in the cow. Theriogenology, v.13, p.141-154, 1980.

CRITSER, J. K.; ROWE, R.F.; DEL CAMPO, M. R.; GINTHER, O. J. Embryo transfer in cattle: factors affecting superovulatory response, number of transferable embryos, and length of post-treatment estrous cycles. Theriogenology, v.13, n.6, p.397406,1980.

DALLA PORTA, M. A. \& BEM, A. R. Aspectos fisiopatológicos do desenvolvimento embrionário (bovino). A Hora Veterinária, v.2, n.8, p.30-36, 1982.

DONALDSON, L. E. Effect of insemination regimen on embryon production in superovulated cows. Vet. Record., v.117, p.35-37, 1985.

DONALDSON, L. E.; WARD, D. N.; GLENN, S. D. Use of porcine follicle stimulating hormone after chromatographic purification in superovulation of cattle. Theriogenology, v.25. n.6, p.747-757, 1986.

ELSDEN, R. P.; HASLER, J.F.; SEIDEL, G. E. J. Nonsurgical recovery of bovine eggs. Theriogenology, v.6, p.523-532, 1976.

FOOTE, R. H. \& ELLIGTON, J. E. Is a superovulated oocyte normal? Theriogenology, v. 29, n.1, p.111123, 1988 .

GREVE, T.; CALLENSEN, H.; HYTTEL, P. Endocrine profiles and egg quality in the superovulated cow. Nord. Vet-Med, v.35, p.408-421, 1983.

GREVE, T.; CALLENSEN, H.; HYTTEL, P. Plasma progesterone profile and embryo quality in superovulated dairy cows. Theriogenology, v.21, n.1, p.238, 1984.

GUIBAULT, L. A.; ROY, G. L.; GRASSO, F.; F.; MENARD, D. P.; BOUSQUET, D. Ovarian follicular dynamics in superovulated heifers pretreated with $\mathrm{FSH}-\mathrm{P}$ at the beginning of the estrous cycle. An ultrassonographic approach. Theriogenology, v.29, n.1, p.257, 1988.

HACKETT, A.J.; DURNFORD, R. J.; MAPLETOFT, R. J.; MARCUS G. J. Location and status of embryos in the genital tract of superovulated cows 4 to 6 days after insemination. Theriogenology, v.40, p.11471153, 1993.

HAHN. J. Recent progress in bovine embryo freezing and transfer in Northern-Germany. In: SYMPOSIUM ON ADVANCED TOPICS IN ANIMAL REPRODUCTION. 2., 1983, Jaboticabal. Proceedings... São Paulo: FCAV-UNESP, 1983. 291p., p.281-284. 
HASLER, J. F.; MCCAULEY, A. D.; SCHERMERHORN E. C.; FOOTE, R. H. Superovulatory responses of holstein cows. Theriogenology, v.19, n.1, p.83-99, 1983.

HAWK, H. W.; CONLEY. H.H.; WALL, R. J.; WHITAKER, R. O. Fertilization rates in superovulating cows after deposition of semen on the infundibulun, near the uterotubal junction or after insemination with high numbers of sperm. Theriogenology, v.29, n.5, p.11311142, 1988.

HERRIER, A,; BECKERS, J. F.; VIVIERS DONNAY, J/ ;; NIEMANN. H. Purified FSH supplemented with defined amounts of $\mathrm{LH}$ for superovulation in dairy cattle. Theriogenology, v.29, n.1, p.260, 1988.

HUNTER, R. H. F. Fertility in cattle: basic reasons why late insemination must be avoided. Animal Breeding Abstracts, v.53, n.2, p.83-87, 1985.

HUNTER, R. H. F. Timing of ovulation in indigenous breedsof cattle in the tropics: experimental methodology for its detection. Animal Breeding Abstracts, v.54, n.7, p.533-538, 1986.

HYTTEL, P.; CALLENSEN, H.; GREVE, T. Ultraestrutural features of preovulatory oocyte maturation in superovulation cattle. J. Reprod. Fert., v.76, p.645-656, 1986.

HYTTEL, P.; GREVE, T.; CALLENSEN, $H$. Ultraestrutural aspects of oocyte maturation and fertilization in cattle, J. Reprod. Fertil. Suppl., v.38, p.35-47, 1989.

KUSAN, F. B. Classification of embryos prior to freezing. In: TECHNIQUES FOR FREEZING MAMMALIAN EMBRYOS: 1986 SHORT COURSE PROCEEDINGS. Fort Collins: Animal Reproduction Laboratory Colorado State University, 1986. p.34.

LOONEY, C. R. Superovulating in beef females. In: ANNUAL CONVENTION OF AMERICAN EMBRYO TRANSFER ASSOCIATION, 5., 1986. Proc. A. E. T. A., 1986, p.16-29.

MAXWELL, P. D., MASSEY, J. M.; KRAEMER, D. C. Timing of ovulations in the superovulated bovine. Theriogenology, v.9, n.1, 1978.

MILLER, D. M.; JOHNSON, W. H.; CATES, W. F.; MAPLETOF, R. J. Superovulation studies in heifers to determine fertilization rates of bulls with high levels of certain sperm defects. Theriogenology, v.15, n.1, p.122, 1981.

MORAES. I.A. Determinação Dos Níveis Plasmáticos de Progesterona Em Fêmeas Bovinas Superovuladas Para um Programa De Transferência De Embriões e Análises Quali-Quantitativa Dos Embriões Obtidos. Niterói, 1991. 48p. Tese (Mestrado) Medicina Veterinária (Patologia Veterinária) - Universidade Federal Fluminense.
MORAES, I. A.; SANTOS, M. R. C.; PINHO, T. G.; RODRIGUES, C. F. M. Progesterona endógena no dia da coleta de embriões bovinos: influência nos aspectos quali-quantitativos. In: Congresso Brasileiro de Medicina Veterinária, 22., 1992, Curitiba, Anais... Curitiba: Associação Bibliotecária do Paraná, 1992, p.073.

SHAMS, D.; SCHALLENBERGER, E.; HOFFMANN, B.; KARG, $H$. The oestrous cycle of the cow: hormonal parameters and time relationships concerning oestrous, ovulation, and eletrical resistance of the vaginal mucus. Acta Endocrinologia, v.86, p.180192, 1977.

SCHIEWE, M. C.; LOONEY, C. R.; JOHNSON, C. A.; HILL, K. G.; GODKE, R. A. Transferable embryo recovery rates following different insemination schedules in superovulated beef cattle. Theriogenology, v.28, n.4, p.395-406, 1987.

SHALLENBERGER, E.; KNOPF. L.; VEH, F. V.; TENHUMBERG, H.; AUMÜLLER, R. Endocrine and ultrasonic evaluation of ovarian response in superovulation indueced by continuous FSH administration, repeated $\mathrm{FSH}$ injections, or PMSG injection. Theriogenology, v.29, n.1, p.302, 1988.

SHEA, B. F. Recovery of bovine follicular oocytes and ther fertilization in a recipient animal. Theriogenology, v.9, n.1, p.101, 1978.

SHI, D. S.; K. H.; GORDON, I. Effects of bulls on fertilization of bovine oocytes and their subsequent development in vitro. Theriogenology, v.33, n.1, p.324, 1990.

SZECHY, M. L. M. Análise do Desempenho de Fêmeas Bovinas da Raça Simental em Programas de Transferência de Embriões. Niterói, 1992, 65p. tese (Mestrado) Medicina Veterinária (Patologia Veterinária) - Universidade Federal Fluminense.

West, G.; West. C.; Risley, D.; DONALDSON, L. Effect of bredding regime on percent ova fertilized in superovulated cows. Theriogenology, v.21, n.1, p.273, 1984.

XU, K. P. \& GREVE, T. A. A detailed analysis of early events during in-vitro fertilization of bovine follicular oocytes. J. Reprod. Fert., v.82, p.127-134, 1988.

YADAV, M. C.; WALTON, J. S.; LESLIE, K. E. Timing of the onset and duration of ovulation in superovulated beef heifers. Theriogenology, v.26, n.4, p.509, 1986.

YAMAMOTO, M.; OOE, M.; FUJII, C.; SUZUKI, T. Superovulation of japaneses Black heifers Treated with FSH-P and FSH-R. J. Vet. Med. Sci., v.55, n.1, p.133-134, 1993. 